Article

\title{
Impulsive Fractional-Like Differential Equations: Practical Stability and Boundedness with Respect to $h$-Manifolds
}

\author{
Gani Stamov ${ }^{1, t, \ddagger}$, Anatoliy Martynyuk ${ }^{2, \ddagger}$ and Ivanka Stamova ${ }^{3, *, \neq}$ \\ Department of Mathematics, Technical University of Sofia, 8800 Sliven, Bulgaria; gstamov@abv.bg \\ 2 S.P. Timoshenko Institute of Mechanics, NAS of Ukraine, 03057 Kiev-57, Ukraine; center@inmech.kiev.ua \\ 3 Department of Mathematics, University of Texas at San Antonio, San Antonio, TX 78249, USA \\ * Correspondence: ivanka.stamova@utsa.edu \\ + Current address: University of Texas at San Antonio, San Antonio, TX 78249, USA. \\ $\ddagger$ These authors contributed equally to this work.
}

Received: 21 September 2019; Accepted: 4 November 2019; Published: 7 November 2019

check for updates

\begin{abstract}
In this paper, an impulsive fractional-like system of differential equations is introduced. The notions of practical stability and boundedness with respect to $h$-manifolds for fractional-like differential equations are generalized to the impulsive case. For the first time in the literature, Lyapunov-like functions and their derivatives with respect to impulsive fractional-like systems are defined. As an application, an impulsive fractional-like system of Lotka-Volterra equations is considered and new criteria for practical exponential stability are proposed. In addition, the uncertain case is also investigated.
\end{abstract}

Keywords: fractional-like derivative; impulses; practical stability; boundedness; $h$-manifolds

MSC: 34A08; 34A37; 34D20; 34D35

\section{Introduction}

Fractional differential systems have attracted the attention of many researchers, due to their generalizations and wide range of applications in science and technologies. See, for example, the books [1-3] for basic results of systems with fractional derivatives of Riemann-Liouville and Caputo types. Parallel to the development of the theory of fractional systems, numerous definitions of fractional derivatives have been introduced, such as an Atangana-Baleanu fractional derivative, Hadamard-type fractional derivative, Riesz-Miller derivative, and Chen-Machado derivative, just to mention a few [4-13]. The papers [14-16] offered a comprehensive overview and classifications of different types of fractional derivatives.

The notion of "conformable fractional derivative" has been introduced recently in [17]. The new defined derivative has been applied by some authors and interesting results about systems of equations involving such derivatives have been published [18-24].

In [25], the notion of "fractional-like derivative" (FLD) has been proposed as more natural and reflects the essence of the new definition of a fractional derivative. The paper [26] presents important notes about the newly introduced derivatives. The key advantages of the new notions are related to facilitating the evaluation of FLDs of compositions of functions. These advantages coupled with some opportunities for applications lead to the necessity of development of this new direction of research [27]. This task has been addressed by several researchers. For example, results on integral estimates as well as practical stability results have been published in $[28,29]$. 
Indeed, the practical stability concept is of great importance in investigating the dynamic of systems contained within particular bounds during a fixed time interval when a state of the system is possible to be even unstable in the classical Lyapunov's sense, but its performance may be sufficient for the practical point of view. There are numerous problems in engineering, chemistry, and science, in general, for the study of which this concept is essential [30-35].

However, the practical stability analysis of systems with FLDs is still in the initial stage. There are many open problems, and this is the main aim of our research. For example, in [29], impulsive perturbations are not considered. It is well known that impulses can significantly affect the stability behavior of a system, and, therefore, considering impulses into systems of differential equations is a very worthwhile research project with potential applications [34,36,37]. Impulsive systems with conformable derivatives have been considered only in [38,39], where some oscillation criteria and inequalities are proposed. However, the above papers did not offer stability results.

More presently, some results on practical stability theory for impulsive fractional differential systems with Caputo fractional derivatives have been presented in $[40,41]$. However, the mentioned studies do not consider FLDs.

On the other hand, the stability with respect to sets or the manifold concept generalizes the idea of stability of a system [42-45]. To the best of our knowledge, practical stability results with respect to manifolds have not been established for fractional-like differential systems under impulsive perturbations.

The above observations motivated us to study practical stability problems for an impulsive system of fractional-like differential equations. In the present paper, we will apply the Lyapunov technique and extend the practical stability results for differential equations with FLDs to the impulsive case. To this end, we elaborate the definition of FLDs of piecewise continuous Lyapunov-type functions. In addition, we extend the practical stability notion and consider practical stability with respect to manifolds defined by functions. Boundedness criteria are also offered. As an application, an impulsive fractional-like Lotka-Volterra system is investigated. Since considering uncertain values of parameters is very important for applications [46-48], but uncertain fractional-like systems have not been studied, we propose stability results for an uncertain impulsive fractional-like Lotka-Volterra model.

The significance of our paper relative to the existing works is in three aspects:

1. FLDs of piecewise continuous Lyapunov-type functions are defined in this paper.

2. Practical stability and boundedness results with respect to a manifold defined by a specific function for a fractional-like impulsive system are proposed. Thus, our research is a starting step in the development of the stability theory of fractional-like impulsive systems. Indeed, considering impulsive perturbations in systems with FLDs is an important issue for the theory and applications.

3. The obtained results are applied to an impulsive fractional-like Lotka-Volterra model. The advantages of considering fractional-like notions will significantly simplify the computational work in the study of such models of population biology. The effect of uncertain parameters on the practical stability behavior of fractional-like Lotka-Volterra models is also investigated.

\section{Preliminaries}

Let $\mathbb{R}_{+}=[0, \infty), \mathbb{R}^{n}$ be the $n$-dimensional Euclidean space with a norm $\|\cdot\|$, and let $t_{0} \in \mathbb{R}_{+}$. According to $[17,25]$, for any $q \in(0,1]$, we consider the $q^{t h}$-order $\operatorname{FLD} \mathcal{D}_{t_{0}}^{q} x(t)$ of a continuous function $x(t):\left[t_{0}, \infty\right) \rightarrow \mathbb{R}$ given as

$$
\mathcal{D}_{t_{0}}^{q} x(t)=\lim \left\{\frac{x\left(t+\theta\left(t-t_{0}\right)^{1-q}\right)-x(t)}{\theta}, \theta \rightarrow 0\right\} .
$$

If $t_{0}=0$, then $\mathcal{D}_{t_{0}}^{q}(x(t))$ has the form [20],

$$
\mathcal{D}_{0}^{q} x(t)=\lim \left\{\frac{x\left(t+\theta t^{1-q}\right)-x(t)}{\theta}, \theta \rightarrow 0\right\} .
$$


In this research, we will study a system of fractional-like differential equations defined as

$$
\left\{\begin{array}{l}
\mathcal{D}_{t_{k}}^{q} x(t)=f(t, x(t)), t \neq t_{k}, k=0,1,2, \ldots \\
\Delta x\left(t_{k}\right)=x\left(t_{k}^{+}\right)-x\left(t_{k}\right)=I_{k}\left(x\left(t_{k}\right)\right), k=1,2, \ldots
\end{array}\right.
$$

where $x \in \mathbb{R}^{n}, f \in C\left(\mathbb{R}_{+} \times \mathbb{R}^{n}, \mathbb{R}^{n}\right), t_{0}<t_{1}<t_{2} \cdots<t_{k}<t_{k+1}<\ldots, \lim _{k \rightarrow \infty} t_{k}=\infty, x\left(t_{k}^{+}\right)=$ $\lim _{h \rightarrow 0^{+}} x\left(t_{k}+h\right), x\left(t_{k}^{-}\right)=x\left(t_{k}\right), I_{k} \in C\left(\mathbb{R}^{n}, \mathbb{R}^{n}\right), k=1,2, \ldots$

To establish the symbol $\mathcal{D}_{t_{k}}^{q} x(t)$ and the system (1), we introduce the next definitions $[38,39]$ :

Definition 1. For given $\tilde{t} \in \mathbb{R}_{+}$and $0<q \leq 1$, the $q^{\text {th }}$-order fractional-like derivative $\mathcal{D}_{\tilde{t}}^{q} x(t)$ for a function $x:[\tilde{t}, \infty) \rightarrow \mathbb{R}^{n}$ is defined as

$$
\mathcal{D}_{\tilde{t}}^{q} x(t)=\lim \left\{\frac{x\left(t+\theta(t-\tilde{t})^{1-q}\right)-x(t)}{\theta}, \theta \rightarrow 0\right\}, t>\tilde{t} .
$$

By definition,

$$
\mathcal{D}_{t_{k}}^{q} x\left(t_{k}\right)=\lim _{t \rightarrow t_{k}^{+}} \mathcal{D}_{t_{k}}^{q} x(t) .
$$

If the fractional-like derivative $\mathcal{D}_{\tilde{t}}^{q} x(t)$ of order $q$ of a continuous function $x(t)$ exists at any point of an open interval of the type $(\tilde{t}, b)$ for some $b>\tilde{t}, t>\tilde{t}, \tilde{t} \in \mathbb{R}_{+}$, then we will say that the function $x(t)$ is $q$-differentiable on $(\tilde{t}, b)$.

Definition 2. System (1) is said to be a system of fractional-like impulsive differential equations.

Let $x_{0} \in \mathbb{R}^{n}$. We will denote the solution of the fractional-like impulsive system (1) that satisfies the initial condition

$$
x\left(t_{0}\right)=x_{0} .
$$

by $x(t)=x\left(t ; t_{0}, x_{0}\right)$.

Note that, according to the second (impulsive) condition in (1) [36,37], the solutions $x(t)$ of type (1) systems are piecewise continuous functions that have points of discontinuity of the first kind $t_{k}$ and are left continuous at these moments. For such functions, the following identities are satisfied:

$$
x\left(t_{k}^{-}\right)=x\left(t_{k}\right), x\left(t_{k}^{+}\right)=x\left(t_{k}\right)+I_{k}\left(x\left(t_{k}\right)\right) .
$$

We will also extend the notion of a fractional-like integral [25], and introduce a fractional-like integral of order $0<q \leq 1$ with a lower limit $\tilde{t}, \tilde{t} \geq 0$, of a function $x:[\tilde{t}, \infty) \rightarrow \mathbb{R}^{n}$ as

$$
I_{\tilde{t}}^{q} x(t)=\int_{\tilde{t}}^{t}(s-\tilde{t})^{q-1} x(s) d s .
$$

As an example, we will consider the next scalar fractional-like impulsive differential equation

$$
\left\{\begin{array}{l}
\mathcal{D}_{t_{k}}^{q} x(t)=-\kappa x(t), t \neq t_{k}, k=0,1,2, \ldots \\
\Delta x\left(t_{k}\right)=\mu_{k} x\left(t_{k}\right), k=1,2, \ldots
\end{array}\right.
$$

where $\kappa>0, \mu_{k} \in \mathbb{R}, k=1,2, \ldots$ 
By direct calculations, using the definition of fractional-like integrals for $t \in\left(t_{k}, t_{k+1}\right]$, we have that the solution of Label (3) satisfies

$$
x(t)=x\left(t_{0}\right) E_{q}\left(-\kappa, t-t_{k}\right) \prod_{l=1}^{k}\left(1+\mu_{l}\right) E_{q}\left(-\kappa, t_{l}-t_{l-1}\right), t_{0} \in \mathbb{R}_{+},
$$

where $E_{q}(\nu, s)$ is the fractional-like exponential function given by $[17,23]$

$$
E_{q}(v, s)=\exp \left(v \frac{s^{q}}{q}\right), v \in \mathbb{R}, s \in \mathbb{R}_{+} .
$$

We will further assume that, for $\left(t_{0}, x_{0}\right) \in \operatorname{int}\left(\mathbb{R}_{+} \times \mathbb{R}^{n}\right)$, the solution $x\left(t ; t_{0}, x_{0}\right)$ of the initial value problem (IVP) (1)-(2) exists on $\left[t_{0}, \infty\right)$. In addition, it is assumed that $f(t, 0)=0, I_{k}(0)=0$ for all $t \geq t_{0}, k=1,2, \ldots$

The following properties of the generalized FLDs $\mathcal{D}_{\tilde{t}}^{q} x(t), t>\tilde{t}$ for some $\tilde{t} \in \mathbb{R}_{+}$can be proved in the same way as the corresponding properties for the FLD $\mathcal{D}_{t_{0}}^{q} x(t), t>t_{0}$ for $t_{0} \in \mathbb{R}_{+}$given in $[17,21,22]$.

Lemma 1. Let $l(y(t)):(\tilde{t}, \infty) \rightarrow \mathbb{R}$. If $l(\cdot)$ is differentiable with respect to $y(t)$ and $y(t)$ is $q$-differentiable on $(\tilde{t}, \infty)$, where $0<q \leq 1$, then for any $t \in \mathbb{R}_{+}, t \neq \tilde{t}$ and $y(t) \neq 0$

$$
\mathcal{D}_{\tilde{t}}^{q} l(y(t))=l^{\prime}(y(t)) \mathcal{D}_{\tilde{t}}^{q} y(t),
$$

where $l^{\prime}$ is a partial derivative of $l(\cdot)$.

Lemma 2. Let the function $x(t):(\tilde{t}, \infty) \rightarrow \mathbb{R}$ be q-differentiable for $0<q \leq 1$. Then, for all $t>\tilde{t}$,

$$
I_{\tilde{t}}^{q}\left(\mathcal{D}_{\tilde{t}}^{q} x(t)\right)=x(t)-x(\tilde{t}) .
$$

Remark 1. For $\tilde{t}=t_{0}$, the definitions of the FLDs and integrals above will be reduced to the definitions in [25]. For more properties of FLDs, we refer the reader to [17-24,26-29].

Let $h:\left[t_{0}, \infty\right) \times \mathbb{R}^{n} \rightarrow \mathbb{R}$ be a continuous function. The next sets will be called $h$-manifolds defined by the function $h$ :

$$
\begin{gathered}
M_{t}(\lambda)=\left\{x \in \mathbb{R}^{n}:|h(t, x)|<\lambda, t \in\left[t_{0}, \infty\right)\right\}, \lambda>0, \\
M_{t}(\bar{\lambda})=\left\{x \in \mathbb{R}^{n}:|h(t, x)| \leq \lambda, t \in\left[t_{0}, \infty\right)\right\} .
\end{gathered}
$$

In the future considerations, we will also assume that the set $M_{t}(\lambda)$ is an $(n-1)$-dimensional manifold in $\mathbb{R}^{n}$.

We will use the following definitions for practical stability of the system (1) with respect to manifolds defined by the function $h$ given in [29].

Definition 3. The fractional-like impulsive system (1) is:

(a) practically stable with respect to the function $h$, if given $(\lambda, A)$ with $0<\lambda<A$, for any $x_{0} \in M_{t_{0}}(\lambda)$ it follows $x\left(t ; t_{0}, x_{0}\right) \in M_{t}(A), t \geq t_{0}$ for some $t_{0} \in \mathbb{R}_{+}$;

(b) uniformly practically stable with respect to the function $h$, if (a) holds for every $t_{0} \in \mathbb{R}_{+}$;

(c) practically exponentially stable with respect to the function $h$, if given $(\lambda, A)$ with $0<\lambda<A$ for any $x_{0} \in M_{t_{0}}(\lambda)$, we have

$$
x\left(t ; t_{0}, x_{0}\right) \in M_{t}\left(A+\gamma\left|h\left(t_{0}, x_{0}\right)\right| E_{q}\left(-\kappa, t-t_{0}\right)\right), t \geq t_{0}, \text { for some } t_{0} \in \mathbb{R}_{+},
$$


where $0<q<1, \gamma, \kappa>0$.

Let $G_{k}=\left(t_{k-1}, t_{k}\right) \times \mathbb{R}^{n}, k=1,2, \ldots, G=\bigcup_{k=1}^{\infty} G_{k}$ and $B_{r}=\left\{x \in \mathbb{R}^{n}:\|x\|<r\right\}, r>0$.

Next, for any $t_{k} \in \mathbb{R}_{+}, k=0,1,2, \ldots$, we will introduce the class $V_{t_{k}}^{q}$ of piecewise continuous auxiliary Lyapunov-type functions that will be used in our research (see, for example, $[36,41]$ and the references therein).

Definition 4. The function $V \in V_{t_{k}}^{q}$, if:

1. $\quad V$ is defined on $G, V$ has nonnegative values and $V(t, 0)=0$ for $t \geq t_{k}$;

2. $V$ is continuous in $G, q$-differentiable in $t$ and locally Lipschitz continuous with respect to its second argument on each of the sets $G_{k}$;

3. For each $k=0,1,2, \ldots$ and $x \in \mathbb{R}^{n}$, there exist the finite limits

$$
V\left(t_{k}^{-}, x\right)=\lim _{\substack{t \rightarrow t_{k} \\ t<t_{k}}} V(t, x), \quad V\left(t_{k}^{+}, x\right)=\lim _{\substack{t \rightarrow t_{k} \\ t>t_{k}}} V(t, x),
$$

and $V\left(t_{k}^{-}, x\right)=V\left(t_{k}, x\right)$.

For a function $V \in V_{t_{k}}^{q}, t>t_{k}$, we define the expressions:

$$
\begin{gathered}
{ }^{+} \mathcal{D}_{t_{k}}^{q} V(t, x) \\
=\limsup \left\{\frac{V\left(t+\theta\left(t-t_{k}\right)^{1-q}, x\left(t+\theta\left(t-t_{k}\right)^{1-q} ; t, x\right)\right)-V(t, x)}{\theta}, \theta \rightarrow 0^{+}\right\},
\end{gathered}
$$

as the upper right fractional-like derivative of the Lyapunov-type function,

$$
\begin{gathered}
+\mathcal{D}_{t_{k}}^{q} V(t, x) \\
=\liminf \left\{\frac{V\left(t+\theta\left(t-t_{k}\right)^{1-q}, x\left(t+\theta\left(t-t_{k}\right)^{1-q} ; t, x\right)\right)-V(t, x)}{\theta}, \theta \rightarrow 0^{+}\right\},
\end{gathered}
$$

as the lower right fractional-like derivative of the Lyapunov-type function,

$$
\begin{aligned}
& { }^{-} \mathcal{D}_{t_{k}}^{q} V(t, x) \\
& =\limsup \left\{\frac{V\left(t+\theta\left(t-t_{k}\right)^{1-q}, x\left(t+\theta\left(t-t_{k}\right)^{1-q} ; t, x\right)\right)-V(t, x)}{\theta}, \theta \rightarrow 0^{-}\right\},
\end{aligned}
$$

as the upper left fractional-like derivative of the Lyapunov-type function,

$$
\begin{gathered}
-\mathcal{D}_{t_{k}}^{q} V(t, x) \\
=\liminf \left\{\frac{V\left(t+\theta\left(t-t_{k}\right)^{1-q}, x\left(t+\theta\left(t-t_{k}\right)^{1-q} ; t, x\right)\right)-V(t, x)}{\theta}, \theta \rightarrow 0^{-}\right\},
\end{gathered}
$$

as the lower left fractional-like derivative of the Lyapunov-type function.

Let $x\left(t ; t_{0}, x_{0}\right)$ be the solution of the IVP (1)-(2), which exists and is defined on $\mathbb{R}_{+} \times B_{r}$. Then, Ref. [25], the fractional-like derivative of the function $V(t, x)$ with respect to the solution $x\left(t ; t_{0}, x_{0}\right)$, is defined by

$$
\begin{gathered}
{ }^{+} \mathcal{D}_{t_{k}}^{q} V(t, x) \\
=\limsup \left\{\frac{V\left(t+\theta\left(t-t_{k}\right)^{1-q}, x+\theta\left(t-t_{k}\right)^{1-q} f(t, x)\right)-V(t, x)}{\theta}, \theta \rightarrow 0^{+}\right\} .
\end{gathered}
$$


If $V(t, x(t))=V(x(t)), 0<q \leq 1, V$ is differentiable on $x$, and $x(t)$ is $q$-differentiable on $t$ for $t>t_{k}$, then

$$
{ }^{+} \mathcal{D}_{t_{k}}^{q} V(t, x)=V^{\prime}(x(t)) \mathcal{D}_{t_{k}}^{q} x(t),
$$

where $V^{\prime}$ is a partial derivative of the function $V$.

From Labels (3) and (5), it follows:

$$
{ }^{+} \mathcal{D}_{t_{k}}^{q} V\left(t, x\left(t ; t_{0}, x_{0}\right)\right)=\left.{ }^{+} \mathcal{D}_{t_{k}}^{q} V(t, x)\right|_{(1)},
$$

$t>t_{k}, k=0,1,2, \ldots$

Furthermore, we will use a comparison result $[29,36]$, and, for this reason together with (1), we consider the comparison equation:

$$
\left\{\begin{array}{l}
\mathcal{D}_{t_{k}}^{q} y(t)=F(t, y), \quad t \neq t_{k}, k=0,1,2, \ldots, \\
\Delta y\left(t_{k}\right)=y\left(t_{k}^{+}\right)-y\left(t_{k}\right)=J_{k}\left(y\left(t_{k}\right)\right), \quad k=1,2, \ldots,
\end{array}\right.
$$

where $F:\left[t_{0}, \infty\right) \times \mathbb{R}_{+} \rightarrow \mathbb{R}_{+}, J_{k}: \mathbb{R}_{+} \rightarrow \mathbb{R}_{+}, k=1,2, \ldots$

Let $y_{0} \in \mathbb{R}_{+}$and $y^{+}(t)=y^{+}\left(t ; t_{0}, y_{0}\right)$ be the maximal solution $[29,36]$ of Equation (6), which satisfies the initial condition $y\left(t_{0}^{+}\right)=y_{0}$.

In what follows, we will consider only nonnegative solutions $y(t)$ of the comparison Equation (6).

Definition 5. Equation (6) is said to be:

(a) practically stable with respect to $(\lambda, A)$, if given $(\lambda, A)$ with $0<\lambda<A$ for any $y_{0}<\lambda$ it follows $y^{+}\left(t ; t_{0}, y_{0}\right)<A, t \geq t_{0}$ for some $t_{0} \in \mathbb{R}_{+} ;$

(b) uniformly practically stable with respect to $(\lambda, A)$, if (a) holds for every $t_{0} \in \mathbb{R}_{+}$;

(c) practically exponentially stable with respect to $(\lambda, A)$, if given $(\lambda, A)$ with $0<\lambda<A$ for any $y_{0}<\lambda$, we have

$$
y^{+}\left(t ; t_{0}, y_{0}\right)<A+\gamma y_{0} E_{q}\left(-\kappa, t-t_{0}\right)
$$

for some $t_{0} \in \mathbb{R}_{+}$, where $0<q<1, \gamma, \kappa>0$.

The proof of the next comparison lemma is similar to the proof of Theorem 5.1 in [25], and we omit it.

Lemma 3. Assume that:

1. The function $F:\left[t_{0}, \infty\right) \times \mathbb{R}_{+} \rightarrow \mathbb{R}_{+}$is continuous in each of the sets $\left(t_{k-1}, t_{k}\right] \times \mathbb{R}_{+}, t_{k}>t_{0}$ and, for $\xi \in \mathbb{R}_{+}$, there exists the finite limit

$$
\lim _{\substack{(t, y) \rightarrow(t, \tilde{\xi}) \\ t>t_{k}}} F(t, y) .
$$

2. The functions $J_{k}$ are continuous in $\mathbb{R}_{+}$and $\psi_{k}(y)=y+J_{k}(y) \geq 0, k=1,2, \ldots$, are non-decreasing in $\mathbb{R}_{+}$.

3. The maximal solution $y^{+}\left(t ; t_{0}, y_{0}\right)$ of $(6)$ is defined on $\left[t_{0}, \infty\right)$ and

$$
y^{+}\left(t_{k}^{+} ; t_{0}, y_{0}\right) \in \mathbb{R}_{+} \text {for all } t_{k}>t_{0}
$$

4. The function $V:\left[t_{0}, \infty\right) \times B_{r} \rightarrow \mathbb{R}_{+}, V \in V_{t_{k}}^{q}$ is such that, for $t \in\left[t_{0}, \infty\right), x \in B_{r}$,

$$
\begin{aligned}
& V\left(t^{+}, x+I_{k}(x)\right) \leq \psi_{k}(V(t, x)), \quad t=t_{k}, \quad k=1,2, \ldots \\
& { }^{+} \mathcal{D}_{t_{k}}^{q} V(t, x) \leq F(t, V(t, x)), \quad t \neq t_{k}, \quad k=0,1,2, \ldots
\end{aligned}
$$


Then, $V\left(t_{0}^{+}, x_{0}\right) \leq y_{0}$ implies

$$
V\left(t, x\left(t ; t_{0}, x_{0}\right)\right) \leq y^{+}\left(t ; t_{0}, y_{0}\right), \quad t \in\left[t_{0}, \infty\right) .
$$

In the next section, we will need the following lemma whose proof is similar to the proofs of corollaries 5.3 and 5.4 in [25] using the generalized definition for FLDs. Similar results for equations with fractional Caputo-type derivatives are given in [36].

Lemma 4. Assume that the function $V \in V_{t_{k}}^{q}$ is such that for $t \in\left[t_{0}, \infty\right), x \in B_{r}$,

$$
\begin{gathered}
V\left(t_{k}^{+}, x\right) \leq V\left(t_{k}, x\right), \quad k=1,2, \ldots, \\
{ }^{+} \mathcal{D}_{t_{k}}^{q} V(t, x) \leq-\kappa V(t, x)+g(t), t \neq t_{k}, k=0,1,2, \ldots,
\end{gathered}
$$

where $\kappa=$ const $>0, g: \mathbb{R} \rightarrow \mathbb{R}_{+}$is continuous.

Then,

$$
\begin{gathered}
V(t, x(t)) \leq V\left(t_{0}^{+}, x_{0}\right) E_{q}\left(-\kappa, t-t_{0}\right)+\int_{t_{k}}^{t} \frac{W^{q}\left(t-t_{k}, s-t_{k}\right) g(s)}{\left(s-t_{k}\right)^{1-q}} d s \\
+\sum_{j=1}^{k} \prod_{l=k-j+1}^{k} E_{q}\left(-\kappa, t_{l}-t_{l-1}\right) \int_{t_{k-j}}^{t_{k-j+1}} \frac{W^{q}\left(t-t_{k} s-t_{k-j}\right) g(s)}{\left(s-t_{k_{j}}\right)^{1-q}} d s, t \geq t_{0},
\end{gathered}
$$

where $W^{q}\left(t-t_{k}, s-t_{k}\right)=E_{q}\left(-\kappa, t-t_{k}\right) E_{q}\left(\kappa, s-t_{k}\right)$.

In addition, we will need the Hahn classes of functions $K=\left\{a \in C\left[\mathbb{R}_{+}, \mathbb{R}_{+}\right]: a(u)\right.$ is strictly increasing and $a(0)=0\}$ and $C K=\left\{a \in C\left[\mathbb{R}_{+}^{2}, \mathbb{R}_{+}\right]: a(t, u) \in K\right.$ for each $t \in \mathbb{R}_{+}$and $a(t, u) \rightarrow \infty$ as $u \rightarrow \infty\}$.

\section{Main Results}

In this section, we will state our main practical stability and boundedness criteria for impulsive systems with FLDs. These results extend and generalize the results in $[29,31-34,36,40,41]$ for different classes of differential, functional differential and fractional differential equations, and are first contributions to the stability theory of impulsive equations with FLDs.

\subsection{Practical Stability Criteria}

Theorem 1. Assume that $0<\lambda<A$ are given, and:

1. Conditions of Lemma 3 are met, and $F(t, 0)=0, J_{k}(0)=0$ for $t \in\left[t_{0}, \infty\right), k=1,2, \ldots$

2. For the function $V(t, x) \in V_{t_{k}}^{q}$, the following condition holds

$$
a(|h(t, x)|) \leq V(t, x) \leq \eta(t) b(|h(t, x)|),(t, x) \in\left[t_{0}, \infty\right) \times \mathbb{R}^{n},
$$

where $a, b \in K$ and the function $\eta(t) \geq 1$ is defined and continuous for $t \in\left[t_{0}, \infty\right)$.

3. $\eta\left(t_{0}\right) b(\lambda)<a(A)$.

Then:

(a) If (6) is practically stable with respect to $\left(\eta\left(t_{0}\right) b(\lambda), a(A)\right)$, then the system (1) is practically stable with respect to the function $h$.

(b) If (6) is uniformly practically stable with respect to $\left(\eta\left(t_{0}\right) b(\lambda), a(A)\right)$, then the system (1) is uniform practically stable with respect to the function $h$.

Proof. (a) From the practical stability of (6) with respect to $\left(\eta\left(t_{0}\right) b(\lambda), a(A)\right)=\left(\lambda^{*}, A^{*}\right)$ and condition 3 of Theorem 1, we have

$$
y_{0}<\lambda^{*} \text { implies } y^{+}\left(t ; t_{0}, y_{0}\right)<A^{*}, t \geq t_{0}
$$


for some given $t_{0} \in \mathbb{R}_{+}$.

Let $x_{0} \in M_{t_{0}}(\lambda)$. Then, we have

$$
\eta\left(t_{0}\right) b\left(\left|h\left(t_{0}, x_{0}\right)\right|\right)<\lambda^{*}
$$

and, from (7), we get

$$
V\left(t_{0}, x_{0}\right) \leq \eta\left(t_{0}\right) b\left(\left|h\left(t_{0}, x_{0}\right)\right|\right)<\lambda^{*} .
$$

Hence, using (8), we obtain

$$
y^{+}\left(t ; t_{0}, V\left(t_{0}, x_{0}\right)\right)<A^{*}
$$

for $t \geq t_{0}$.

From Lemma 3, for the solution of the IVP (1), (2) $x(t)=x\left(t ; t_{0}, x_{0}\right)$, we get

$$
V\left(t, x\left(t ; t_{0}, x_{0}\right)\right) \leq y^{+}\left(t ; t_{0}, V\left(t_{0}, x_{0}\right)\right), t \in\left[t_{0}, \infty\right) .
$$

From (7), (9) and (10), there follow the inequalities:

$$
a\left(\left|h\left(t, x\left(t ; t_{0}, x_{0}\right)\right)\right|\right) \leq V\left(t, x\left(t ; t_{0}, x_{0}\right)\right) \leq y^{+}\left(t ; t_{0}, V\left(t_{0}, x_{0}\right)\right)<a(A), t \geq t_{0} .
$$

Hence, $\left|h\left(t, x\left(t ; t_{0}, x_{0}\right)\right)\right|<A$ for $t \geq t_{0}$, which proves the practical stability of (1) with respect to the function $h$.

(b) The proof of this section can be conducted analogous to the proof of section (a). In this case, it is possible to choose $\lambda$ and $\lambda^{*}$ that do not depend on $t_{0}$.

The proof of Theorem 1 is complete.

The proof of the next theorem can be obtained via arguments analogous to the ones in Theorem 1. To study the uniform practical stability properties of the impulsive fractional-like system (1), we apply functions from the class $C K$.

Theorem 2. Assume that $0<\lambda<A$ are given, and:

1. Condition 1 of Theorem 1 holds.

2. There exist functions $V(t, x) \in V_{t_{k^{\prime}}}^{q} a \in K$ and $b \in C K$ such that

$$
a(|h(t, x)|) \leq V(t, x) \leq b(t,|h(t, x)|),(t, x) \in\left[t_{0}, \infty\right) \times \mathbb{R}^{n} .
$$

3. $b\left(t_{0}, \lambda\right)<a(A)$.

Then, the uniform practical stability with respect to $\left(b\left(t_{0}, \lambda\right), a(A)\right)$ of $(6)$ implies uniform practical stability of the system (1) with respect to the function $h$.

Theorem 3. If in Theorem 1, instead of condition (7), we have

$$
|h(t, x)| \leq V(t, x) \leq b(t,|h(t, x)|),(t, x) \in\left[t_{0}, \infty\right) \times \mathbb{R}^{n},
$$

where $b \in C K$, and $b\left(t_{0}, \lambda\right)<\left|h\left(t_{0}, x_{0}\right)\right|$, then the practical exponential stability of (6) with respect to $(\lambda, A)$ implies the practical exponential stability of the system (1) with respect to the function $h$.

Proof. Let $0<\lambda<A$. If (6) is practically exponentially stable with respect to $(\lambda, A)$, then, for any $y_{0}<\lambda$, we have

$$
y^{+}\left(t ; t_{0}, y_{0}\right)<A+\gamma y_{0} E_{q}\left(-\kappa, t-t_{0}\right), t \geq t_{0}
$$

for some $t_{0} \in \mathbb{R}_{+}$, where $\gamma>0, \kappa>0$.

Let $x_{0} \in M_{t_{0}}(\lambda)$. From Lemma 3, for the solution of the IVP (1), (2) $x(t)=x\left(t ; t_{0}, x_{0}\right)$ for $y_{0}=V\left(t_{0}, x_{0}\right)$, we get (10). 
From (11) and (10), we obtain

$$
\begin{gathered}
\left|h\left(t, x\left(t ; t_{0}, x_{0}\right)\right)\right| \leq V\left(t, x\left(t ; t_{0}, x_{0}\right)\right) \leq y^{+}\left(t ; t_{0}, V\left(t_{0}, x_{0}\right)\right) \\
<A+\gamma b\left(t_{0}, \lambda\right) E_{q}\left(-\kappa, t-t_{0}\right), t \geq t_{0} .
\end{gathered}
$$

Therefore,

$$
x\left(t ; t_{0}, x_{0}\right) \in M_{t}\left(A+\gamma\left|h\left(t_{0}, x_{0}\right)\right| E_{q}\left(-\kappa, t-t_{0}\right)\right)
$$

for $t \geq t_{0}, 0<q \leq 1$, which proves the practical exponential stability of (1) with respect to the function $h$.

Corollary 1. Assume that $0<\lambda<A$ are given, and:

1. Conditions of Lemma 4 hold for $g(t) \equiv 0, t \in\left[t_{0}, \infty\right)$.

2. For the function $V(t, x) \in V_{t_{k}}^{q}$ the following condition holds

$$
|h(t, x)|-A<V(t, x) \leq \Lambda(r)|h(t, x)|,(t, x) \in\left[t_{0}, \infty\right) \times \mathbb{R}^{n},
$$

where the function $\Lambda(r) \geq 1$ is defined and continuous for any $0<r \leq \infty$.

Then, system (1) is practically exponentially stable with respect to the function $h$.

Proof. Let $t_{0} \in \mathbb{R}_{+}$. For the function $V(t, x)$ and any values of $0<q \leq 1$, we deduce from Lemma 4

$$
V(t, x(t)) \leq V\left(t_{0}, x_{0}\right) E_{q}\left(-\kappa, t-t_{0}\right), t \geq t_{0}
$$

From (12) and (13), we have

$$
\begin{gathered}
\left|h\left(t, x\left(t ; t_{0}, x_{0}\right)\right)\right|-A<V\left(t, x\left(t ; t_{0}, x_{0}\right)\right) \leq V\left(t_{0}, x_{0}\right) E_{q}\left(-\kappa, t-t_{0}\right) \\
\leq \Lambda(r)\left|h\left(t_{0}, x_{0}\right)\right| E_{q}\left(-\kappa, t-t_{0}\right), t \geq t_{0} .
\end{gathered}
$$

Therefore,

$$
x\left(t ; t_{0}, x_{0}\right) \in M_{t}\left(A+\gamma_{1}\left|h\left(t_{0}, x_{0}\right)\right| E_{q}\left(-\kappa, t-t_{0}\right)\right),
$$

where $\gamma_{1}=$ const $>\Lambda(r)$ for any $0<r \leq \infty$. Then, (1) is practically exponentially stable with respect to the function $h$.

\subsection{Boundedness Results}

In this section, we will state our boundedness results for systems of differential equations of the type (1) with fractional-like derivatives. Note that boundedness results for fractional differential equations are very rare [49] in the existing literature. To the best of our knowledge, there has not been any work so far dedicated to investigation of the boundedness properties of a system of differential equations with FLDs.

We shall use the following boundedness definitions [29].

Definition 6. We say that the solutions of system (1) are:

(a) equi-bounded with respect to the function $h$, if

$$
\begin{gathered}
\left(\forall t_{0} \in \mathbb{R}_{+}\right)(\forall \alpha>0)\left(\exists \beta=\beta\left(t_{0}, \alpha\right)>0\right)\left(\forall x_{0} \in M_{t_{0}}(\bar{\alpha})\right) \\
\left(\forall t \geq t_{0}\right): x\left(t ; t_{0}, x_{0}\right) \in M_{t}(\beta) ;
\end{gathered}
$$

(b) uniformly bounded with respect to the function $h$, if the number $\beta$ in (a) is independent of $t_{0} \in \mathbb{R}_{+}$; 
(c) ultimately bounded with respect to the function $h$ for bound $N$, if

$$
\begin{gathered}
(\exists N>0)\left(\forall t_{0} \in \mathbb{R}_{+}\right)(\forall \alpha>0)\left(\exists T=T\left(t_{0}, \alpha\right)>0\right)\left(\forall x_{0} \in M_{t_{0}}(\bar{\alpha})\right) \\
\left(\forall t \geq t_{0}+T\right): x\left(t ; t_{0}, x_{0}\right) \in M_{t}(N) ;
\end{gathered}
$$

(d) uniformly ultimately bounded with respect to the function $h$ for bound $N$, if the number $T$ from (c) does not depend on $t_{0} \in \mathbb{R}_{+}$.

In the next, boundedness properties of the positive solutions of (6) are defined as follows.

Definition 7. We say that the solutions of (6) are:

(a) equi-bounded, if

$$
\begin{gathered}
\left(\forall t_{0} \in \mathbb{R}_{+}\right)(\forall \alpha>0)\left(\exists \beta=\beta\left(t_{0}, \alpha\right)>0\right)\left(\forall y_{0} \in \mathbb{R}_{+}: y_{0} \leq \alpha\right) \\
\left(\forall t \geq t_{0}\right): y^{+}\left(t ; t_{0}, y_{0}\right)<\beta ;
\end{gathered}
$$

(b) uniformly bounded, if the number $\beta$ in (a) is independent of $t_{0} \in \mathbb{R}_{+}$;

(c) ultimately bounded for bound $N$, if

$$
\begin{gathered}
(\exists N>0)\left(\forall t_{0} \in \mathbb{R}_{+}\right)(\forall \alpha>0)\left(\exists T=T\left(t_{0}, \alpha\right)>0\right)\left(\forall y_{0} \in \mathbb{R}_{+}: y_{0} \leq \alpha\right) \\
\left(\forall t \geq t_{0}+T\right): y^{+}\left(t ; t_{0}, y_{0}\right)<N
\end{gathered}
$$

(d) uniformly ultimately bounded for bound $N$, if the number $T$ from (c) does not depend on $t_{0} \in \mathbb{R}_{+}$.

Theorem 4. Assume that conditions of Theorem 1 hold and $a(u) \rightarrow \infty$ as $u \rightarrow \infty$. Then,

(a) If the solutions of (6) are equi-bounded, then the solutions of system (1) are equi-bounded with respect to the function $h$.

(b) If the solutions of (6) are ultimately bounded for a bound $N$, then the zero solution of system (1) are ultimately bounded for the bound $a^{-1}(N)$ with respect to the function $h$.

Proof. (a) Let $t_{0} \in \mathbb{R}_{+}$and $\alpha>0$ be given. Set $\alpha^{*}=\eta\left(t_{0}\right) b(\alpha)$. Then, $a(u) \rightarrow \infty$ as $u \rightarrow \infty$, implies $\alpha \rightarrow \infty$ as $\alpha^{*} \rightarrow \infty$.

The equi-boundedness of the solutions of (6) implies the existense of a $\beta_{1}=\beta_{1}\left(t_{0}, \alpha\right)$ such that, for any $y_{0} \in \mathbb{R}_{+}$with $y_{0} \leq \alpha^{*}$, we have

$$
y^{+}\left(t ; t_{0}, y_{0}\right)<\beta_{1}, t \geq t_{0}
$$

We denote

$$
\beta=\beta\left(t_{0}, \alpha\right)=a^{-1}\left(\beta_{1}\left(t_{0}, \alpha\right)\right) .
$$

Let $x_{0} \in M_{t_{0}}(\bar{\alpha})$. Then, $\eta\left(t_{0}\right) b\left(\left|h\left(t_{0}, x_{0}\right)\right|\right) \leq \alpha^{*}$ and, since

$$
V\left(t_{0}, x_{0}\right) \leq \eta\left(t_{0}\right) b\left(\left|h\left(t_{0}, x_{0}\right)\right|\right),
$$

we have

$$
V\left(t_{0}, x_{0}\right) \leq \alpha^{*}
$$

Hence,

$$
y^{+}\left(t ; t_{0}, V\left(t_{0}, x_{0}\right)\right)<\beta_{1}, t \geq t_{0} .
$$

From (7), (10) and (14), for the solution $x(t)=x\left(t ; t_{0}, x_{0}\right)$ of the IVP (1), (2), we obtain

$$
a\left(\left|h\left(t, x\left(t ; t_{0}, x_{0}\right)\right)\right|\right) \leq V\left(t, x\left(t ; t_{0}, x_{0}\right)\right) \leq y^{+}\left(t ; t_{0}, V\left(t_{0}, x_{0}\right)\right)<\beta_{1}, t \geq t_{0} .
$$


Therefore, $\left|h\left(t, x\left(t ; t_{0}, x_{0}\right)\right)\right|<a^{-1}\left(\beta_{1}\right)=\beta$ for $t \geq t_{0}$, which proves the equi-boundedness of the solutions of (1) with respect to the function $h$.

(b) Let $t_{0} \in \mathbb{R}_{+}, N>0$ and $A>0$ be given. Set again $\alpha^{*}=\eta\left(t_{0}\right) b(\alpha)$. From the ultimate boundedness of the solutions of (6) for a bound $N$, it follows the existence of $T=T\left(t_{0}, \alpha\right)>0$ such that $y_{0} \in \mathbb{R}_{+}$and $y_{0} \leq \alpha^{*}$ imply

$$
y^{+}\left(t ; t_{0}, y_{0}\right)<N, t \geq t_{0}+T .
$$

Let $x_{0} \in M_{t_{0}}(\bar{\alpha})$. Then, we have that

$$
\eta\left(t_{0}\right) b\left(\left|h\left(t_{0}, x_{0}\right)\right|\right) \leq \alpha^{*}
$$

and, since

$$
V\left(t_{0}, x_{0}\right) \leq \eta\left(t_{0}\right) b\left(\left|h\left(t_{0}, x_{0}\right)\right|\right)
$$

we obtain

$$
V\left(t_{0}, x_{0}\right) \leq \alpha^{*}
$$

Hence,

$$
y^{+}\left(t ; t_{0}, V\left(t_{0}, x_{0}\right)\right)<N, t \geq t_{0}+T .
$$

Form (7), (10) and (15), for the solution $x(t)=x\left(t ; t_{0}, x_{0}\right)$ of the IVP (1), (2), we have

$$
a\left(\left|h\left(t, x\left(t ; t_{0}, x_{0}\right)\right)\right|\right) \leq V\left(t, x\left(t ; t_{0}, x_{0}\right)\right) \leq y^{+}\left(t ; t_{0}, V\left(t_{0}, x_{0}\right)\right)<N, t \geq t_{0} .
$$

Therefore, $\left|h\left(t, x\left(t ; t_{0}, x_{0}\right)\right)\right|<a^{-1}(N)$ for $t \geq t_{0}+T$, which proves the ultimate boundedness of the solutions of (1) with respect to the function $h$.

Theorem 5. Assume that conditions of Theorem 2 hold and $a(u) \rightarrow \infty$ as $u \rightarrow \infty$.

Then:

(a) The uniform boundedness of the solutions of (6) implies uniform boundedness of the solutions of system (1) with respect to the function $h$.

(b) The ultimate uniform boundedness of the solutions of (6) implies ultimate uniform boundedness for the bound $a^{-1}(N)$ of the solutions of system (1) with respect to the function $h$.

The proof of Theorem 5 can be done by making use of identical reasonings as the ones seen in the proof of Theorem 4. In this case, we can choose $\beta$ and $T$ to be independent of $t_{0}$.

\section{Applications}

The main goal of the application section is to investigate in the light of practical stability of $h$-manifolds the following system of Lotka-Volterra impulsive fractional-like differential equations:

$$
\left\{\begin{array}{l}
\mathcal{D}_{t_{k}}^{q} u_{i}(t)=u_{i}(t)\left[r_{i}(t)-\sum_{j=1}^{n} a_{i j}(t) u_{j}(t)\right], t \neq t_{k}, k=0,1,2, \ldots, \\
u_{i}\left(t_{k}^{+}\right)=u_{i}\left(t_{k}\right)+P_{i k}\left(u_{i}\left(t_{k}\right)\right), k=1,2, \ldots \\
u_{i 0}=u_{i}\left(t_{0}\right)
\end{array}\right.
$$

where $t_{0} \in \mathbb{R}_{+}, n \geq 2$ is the number of the species, the system's parameters $r_{i}, a_{i j}$ are positive and continuous on $\mathbb{R}_{+}$, and the impulsive functions $P_{i k}$ are continuous on $\mathbb{R}_{+}, i, j=1,2, \ldots, n, k=1,2, \ldots$

Indeed, due to the great opportunities for applications, Lotka-Volterra and related systems have been largely investigated in the literature [50-52], including impulsive models of Lotka-Volterra 
type [50,53-55], as well as fractional Lotka-Volterra models involving Caputo fractional derivatives [56,57]. However, to the best of our knowledge, results on fractional-like systems of Lotka-Volterra type do not exist in the literature.

We note that $[34,36,37,57]$ the solutions $u(t)=\left(u_{1}(t), u_{2}(t), \ldots, u_{n}(t)\right)^{T}$ of the problem $(16)$ are, in general, piecewise continuous functions with points of discontinuity at the moments $t_{k}, k=1,2, \ldots$

In addition, we assume that the solutions of (16) are nonnegative, and, if $u_{i 0}>0$ for some $i$, then $u_{i}(t)>0$ for all $t \geq t_{0}$. If, moreover, $u_{i}\left(t_{k}\right)>0$, then $u_{i}\left(t_{k}\right)+P_{i k}\left(u_{i}\left(t_{k}\right)\right)>0$ for all $i=1,2, \ldots, \mathcal{N}$ and $k=1,2, \ldots$. Note that these assumptions are natural from the applicability point of view. It follows then [50-52] that, for any closed interval contained in $\left(t_{k}, t_{k+1}\right], k=0,1,2, \ldots$, there exist positive numbers $R_{1}$ and $R_{2}$ such that $R_{1} \leq u_{i}(t) \leq R_{2}$ for $i=1,2, \ldots, n$.

We will prove the next theorem.

Theorem 6. Assume that $0<\lambda<A$ are given, and:

1. For $t \in\left[t_{0}, \infty\right)$ for the functions $a_{i j}$, there exists a positive number $\kappa^{*}$ such that

$$
\kappa^{*}<\frac{R_{1}\left(1+R_{1}\right)}{1+R_{2}} \sum_{j=1}^{n} a_{j i}(t), i=1,2, \ldots, n,
$$

and the system's parameters satisfy

$$
\begin{gathered}
G(t)=\sum_{i=1}^{n} \int_{t_{0}}^{\infty} \frac{W^{q}\left(t-t_{k}, s-t_{k}\right)}{\left(s-t_{0}\right)^{1-q}} \bar{r}_{i}(s) d s \\
+\sum_{j=1}^{k} \prod_{l=k-j+1}^{k} E_{q}\left(-\kappa, t_{l}-t_{l-1}\right) \sum_{i=1}^{n} \int_{t_{k-j}}^{t_{k-j+1}} \frac{W^{q}\left(t-t_{k}, s-t_{k-j}\right)}{\left(s-t_{k_{j}}\right)^{1-q}} \bar{r}_{i}(s) d s<\infty,
\end{gathered}
$$

where $\bar{r}_{i}=\frac{R_{2}}{1+R_{1}} r_{i}, i=1,2, \ldots, n$.

2. The functions $P_{i k}$ are such that

$$
P_{i k}\left(u_{i}\left(t_{k}\right)\right)=-\gamma_{i k} u_{i}\left(t_{k}\right), \quad 0<\gamma_{i k}<1, i=1,2, \ldots n, k=1,2, \ldots
$$

3. There exists a function $h(t, u)$ such that

$$
|h(t, u)|-A<\sum_{i=1}^{n} \ln \left(1+u_{i}(t)\right) \leq \Lambda(r)|h(t, u)|, \quad t \in\left[t_{0}, \infty\right),
$$

where $\Lambda(r) \geq 1$ exists for any $0<r \leq \infty$.

Then, (16) is practically exponentially stable with respect to the function $h$.

Proof. Let we suppose, without loss generality, $1<\lambda<G(t)<A$ and let

$$
u(t)=\left(u_{1}(t), u_{2}(t), \ldots, u_{n}(t)\right)^{T}
$$

be any solution of (16).

We define a Lyapunov function

$$
V(u(t))=\sum_{i=1}^{n} \ln \left(1+u_{i}(t)\right) .
$$


Obviously, $V \in V_{t_{k}}^{q}$ and, for $t_{k}>t_{0} \geq 0, k=1,2, \ldots$, from condition 2 of the theorem, we get

$$
V\left(u\left(t_{k}^{+}\right)\right)=\sum_{i=1}^{n} \ln \left(1+u_{i}\left(t_{k}^{+}\right)\right)=\sum_{i=1}^{n} \ln \left[1+\left(1-\gamma_{i k}\right) u_{i}\left(t_{k}\right)\right] \leq V\left(u\left(t_{k}\right)\right) .
$$

On the other hand, for $t \in\left(t_{k}, t_{k+1}\right], k=0,1,2, \ldots$, along (16), we get

$$
\begin{gathered}
{ }^{+} \mathcal{D}_{t_{k}}^{q} V(u(t)) \leq \sum_{i=1}^{n} \frac{1}{1+u_{i}(t)} \mathcal{D}_{t_{k}}^{q} u_{i}(t) \leq \frac{R_{2}}{1+R_{1}} \sum_{i=1}^{n} r_{i}(t)-\frac{R_{1}}{1+R_{2}} \sum_{i=1}^{n} \sum_{j=1}^{n} a_{i j}(t) u_{j}(t) \\
\leq-\kappa^{*} \sum_{j=1}^{n} \ln \left(1+u_{j}(t)\right)+\sum_{i=1}^{n} \bar{r}_{i}(t)=-\kappa^{*} V(u(t))+\sum_{i=1}^{n} \bar{r}_{i}(t) .
\end{gathered}
$$

The last inequality, (17) and Lemma 4 give us

$$
\begin{aligned}
& V(u(t)) \leq V\left(u\left(t_{0}^{+}\right)\right) E_{q}\left(-\kappa^{*}, t-t_{0}\right)+\sum_{i=1}^{n} \int_{t_{k}}^{t} \frac{W^{q}\left(t-t_{k}, s-t_{k}\right)}{\left(s-t_{k}\right)^{1-q}} \bar{r}_{i}(s) d s \\
& +\sum_{j=1}^{k} \prod_{l=k-j+1}^{k} E_{q}\left(-\kappa, t_{l}-t_{l-1}\right) \sum_{i=1}^{n} \int_{t_{k-j}}^{t_{k-j+1}} \frac{W^{q}\left(t-t_{k}, s-t_{k-j}\right)}{\left(s-t_{k_{j}}\right)^{1-q}} \bar{r}_{i}(s) d s .
\end{aligned}
$$

Let now $\left|h\left(t_{0}, u_{0}\right)\right|<\lambda$. Then, from (18) and condition 3 of the theorem, we get

$$
\left|h\left(t, u\left(t ; t_{0}, u_{0}\right)\right)\right|-A<V\left(u\left(t ; t_{0}, u_{0}\right)\right) \leq \Lambda(r)\left|h\left(t_{0}, u_{0}\right)\right| E_{q}\left(-\kappa^{*}, t-t_{0}\right), \quad t \geq t_{0} .
$$

Hence,

$$
u\left(t ; t_{0}, u_{0}\right) \in M_{t}\left(A+\Lambda(r)\left|h\left(t_{0}, u_{0}\right)\right| E_{q}\left(-\kappa^{*}, t-t_{0}\right)\right)
$$

for $t \geq t_{0}$, i.e., system (16) is practically exponentially stable with respect to the function $h$.

Remark 2. Theorem 6 offers sufficient conditions for practical exponential stability with respect to a function for a fractional-like Lotka-Volterra model. Thus, we extend and improve the existing theory and previous works on Lotka-Volterra and related models in population biology to the fractional-like case. Indeed, the recent studies and experiments on fractional systems indicated that fractional models are more effective than integer-order models in numerous applications mainly because of their nonlocal properties [1-13]. In addition, the FLDs have important advantages in computational aspects than classical fractional derivatives, such as Caputo or Riemann-Liouville types [17-29], which make them more appropriate for applications.

Now, we will consider the corresponding to the (16) uncertain case, i.e., we will consider an impulsive system of differential equations with FLDs and uncertain parameters given by

$$
\left\{\begin{array}{l}
\mathcal{D}_{t_{k}}^{q} u_{i}(t)=u_{i}(t)\left[r_{i}(t)+\tilde{r}_{i}(t)-\sum_{j=1}^{n}\left(a_{i j}(t)+\tilde{a}_{i j}(t)\right) u_{j}(t)\right], t \neq t_{k}, k=0,1,2, \ldots, \\
u_{i}\left(t_{k}^{+}\right)=u_{i}\left(t_{k}\right)-\gamma_{i k} u_{i}\left(t_{k}\right)-\tilde{\gamma}_{i k} u_{i}\left(t_{k}\right), k=1,2, \ldots
\end{array}\right.
$$

where the functions $\tilde{r}_{i}, \tilde{a}_{i j} \in C\left[\mathbb{R}_{+}, \mathbb{R}_{+}\right], i, j=1, \ldots, n, k=1,2, \ldots$ and constants $\tilde{\gamma}_{i k}, i, j=$ $1,2, \ldots, n, k=1,2, \ldots$, represent the uncertainty of the system. In the case when all of these uncertain functions and constants are zeros, then we will receive the "nominal system" $(16)[46,48]$.

Definition 8. System (16) is called practically robustly exponentially stable with respect to the function $h$ if for $t_{0} \in \mathbb{R}_{+}, u_{0} \in M_{t_{0}}(\lambda)$ and for any $\tilde{r}_{i}, \tilde{a}_{i j}, \tilde{\gamma}_{i k}, i, j=1, \ldots, n$ the system (19) is practically exponentially stable with respect to the function $h$. 
The proof of the next theorem follows directly from Theorem 6 .

Theorem 7. Assume that:

1. The conditions of Theorem 6 hold.

2. The functions $\tilde{r}_{i}(t), \tilde{a}_{i j}(t)$ are bounded,

$$
\kappa^{*}<\frac{R_{1}\left(1+R_{1}\right)}{1+R_{2}} \sum_{j=1}^{n}\left(a_{j i}(t)+\tilde{a}_{j i}(t)\right), i=1,2, \ldots, n,
$$

and

$$
\begin{gathered}
\tilde{G}(t)=\sum_{i=1}^{n} \int_{t_{0}}^{\infty} \frac{W^{q}\left(t-t_{k}, s-t_{k}\right)}{\left(s-t_{0}\right)^{1-q}}\left(\bar{r}_{i}(s)+\overline{\tilde{r}}_{i}(s)\right) d s \\
+\sum_{j=1}^{k} \prod_{l=k-j+1}^{k} E_{q}\left(-\kappa, t_{l}-t_{l-1}\right) \sum_{i=1}^{n} \int_{t_{k-j}}^{t_{k-j+1}} \frac{W^{q}\left(t-t_{k}, s-t_{k-j}\right)}{\left(s-t_{k_{j}}\right)^{1-q}}\left(\bar{r}_{i}(s)+\bar{r}_{i}(s)\right) d s<\infty,
\end{gathered}
$$

$\overline{\tilde{r}}_{i}=\frac{R_{2}}{1+R_{1}} \tilde{r}_{i}, i=1,2, \ldots, n$.

3. The unknown constants $\tilde{\gamma}_{i k}$ are such that $0<\tilde{\gamma}_{i k}<1-\gamma_{i k}, i=1,2, \ldots, n, k=1,2, \ldots$

Then, system (16) is practically robustly exponentially stable with respect to the function $h$.

\section{Conclusions}

The FLDs have been proposed in order to overcome some difficulties in evaluating fractional derivatives of some classes of functions. With this research, we contribute to the development of the theory of equations with FLDs. In this paper, we extend the concept of fractional-like derivatives of Lyapunov-type functions for the impulsive case. Using the extended concept, a practical stability analysis with respect to manifolds is conducted for impulsive fractional-like systems. The important novelty of our paper is that it offers the first practical stability and boundedness results for such systems. In addition, the obtained results are applied to a fractional-like impulsive system of Lotka-Volterra type. The effects of uncertain terms are also studied. The proposed technique can be applied in the investigation of other fractional-like impulsive models of diverse interest.

Author Contributions: Conceptualization, G.S. and I.S.; methodology, G.S., I.S., and A.M.; formal analysis, G.S., I.S., and A.M.; investigation, G.S., I.S., and A.M.; writing-original draft preparation, I.S.

Funding: This research received no external funding.

Conflicts of Interest: The authors declare no conflict of interest.

\section{References}

1. Kilbas, A.A.; Srivastava, H.M.; Trujillo, J.J. Theory and Applications of Fractional Differential Equations, 1st ed.; Elsevier Science B.V: Amsterdam, The Netherlands, 2006; ISBN 0444518320.

2. Podlubny, I. Fractional Differential Equations, 1st ed.; Academic Press: San Diego, CA, USA, 1999.

3. Cattani, C.; Srivastava, H.M.; Yang, X.-J. (Eds.) Fractional Dynamics, 1st ed.; De Gryuter: Berlin, Germany, 2015; ISBN 978-3-11-047209-7.

4. Ahmad, B.; Alsaedi, A.; Ntouyas, S.K.; Tariboon, J. Hadamard-Type Fractional Differential Equations, Inclusions and Inequalities, 1st ed.; Springer: Cham, Switzerland, 2017; ISBN 978-3-319-84831-0, 978-3-319-52141-1.

5. Al-Ghafri, K.S.; Rezazadeh, H. Solitons and other solutions of $(3+1)$-dimensional space-time fractional modified KdV-Zakharov-Kuznetsov equation. Appl. Math. Nonlinear Sci. 2019, 4, 289-304. [CrossRef]

6. Atangana, A.; Gómez-Aguilar, J.F. Numerical approximation of Riemann-Liouville definition of fractional derivative: From Riemann-Liouville to Atangana-Baleanu. Numer. Methods Partial. Differ. Equ. 2018, 34, 1502-1523. [CrossRef]

7. Baleanu, D.; Diethelm, K.; Scalas, E.; Trujillo, J.J. Fractional Calculus: Models and Numerical Methods, 2nd ed.; World Scientific: Singapore, 2016; ISBN 9813140038. 
8. Bayın, S.S. Definition of the Riesz derivative and its application to space fractional quantum mechanics. J. Math. Phys. 2016, 57, 123501. [CrossRef]

9. Gao, W.; Ghanbari, B.; Baskonus, H.M. New numerical simulations for some real world problems with Atangana-Baleanu fractional derivative. Chaos Solitons Fractals 2019, 128, 34-43. [CrossRef]

10. Jarad, F.; Abdeljawad, T.; Hammouch, Z. On a class of ordinary differential equations in the frame of Atangana-Baleanu fractional derivative. Chaos Solitons Fractals 2018, 117, 16-20. [CrossRef]

11. Morales-Delgado, V.F.; Gómez-Aguilar, J.F.; Saad, K.M.; Khan, M.A.; Agarwal, P. Analytic solution for oxygen diffusion from capillary to tissues involving external force effects: A fractional calculus approach. Physica A 2019, 523, 48-65. [CrossRef]

12. Saqib, M.; Khan, I.; Shafie, S. Application of Atangana-Baleanu fractional derivative to MHD channel flow of CMC-based-CNT's nanofluid through a porous medium. Chaos Solitons Fractals 2018, 116, 79-85. [CrossRef]

13. Taneco-Heránndez, M.A.; Morales-Delgado, V.F.; Gómez-Aguilar, J.F. Fractional Kuramoto-Sivashinsky equation with power law and stretched Mittag-Leffler kernel. Physica A 2019, 527, 121085. [CrossRef]

14. De Oliveira, E.C.; Tenreiro Machado, J.A. A review of definitions for fractional derivatives and integral. Math. Probl. Eng. 2014, 2014, 238459. [CrossRef]

15. Ortigueira, M.D.; Tenreiro Machado, J.A. What is a fractional derivative? J. Comput. Phys. 2015, $293,4-13$. [CrossRef]

16. Ortigueira, M.; Machado, J. Which Derivative? Fractal Fract. 2017, 1, 3. [CrossRef]

17. Abdeljawad, T. On conformable fractional calculus. J. Comput. Appl. Math. 2015, 279, 57-66. [CrossRef]

18. Anderson, D.R.; Ulness, D.J. Properties of the Katugampola fractional derivative with potential application in quantum mechanics. J. Math. Phys. 2015, 56, 063502. [CrossRef]

19. Eslami, M.; Rezazadeh, H. The first integral method for Wu-Zhang system with conformable time-fractional derivative. Calcolo 2016, 53, 475-485. [CrossRef]

20. Katugampola, U. A new fractional derivative with classical properties. arXiv 2014, arXiv:1410.6535.

21. Khalil, R.; Al Horani, M.; Yousef, A.; Sababheh, M. A new definition of fractional derivative. J. Comput. Appl. Math. 2014, 264, 65-70. [CrossRef]

22. Pospíšil, M.; Pospíšilova S̆kripkova, L. Sturm's theorems for conformable fractional differential equation. Math. Commun. 2016, 21, 273-281.

23. Souahi, A.; Ben Makhlouf, A.; Hammami, M.A. Stability analysis of conformable fractional-order nonlinear systems. Indag. Math. 2017, 28, 1265-1274. [CrossRef]

24. Yel, G.; Baskonus, H.M. Solitons in conformable time-fractional Wu-Zhang system arising in coastal design. Pramana J. Phys. 2019, 93, 57. [CrossRef]

25. Martynyuk, A.A.; Stamova, I.M. Fractional-like derivative of Lyapunov-type functions and applications to the stability analysis of motion. Electron. J. Differ. Equ. 2018, 2018, 1-12.

26. Kiskinov, H.; Petkova, M.; Zahariev, A. Remarks about the existence of conformable derivatives and some consequences. arXiv 2019, arXiv:1907.03486.

27. Martynyuk, A.A. On the stability of the solutions of fractional-like equations of perturbed motion. Dopov. Nats. Akad. Nauk Ukr. Mat. Prirodozn. Tekh. Nauki 2018, 6, 9-16. (In Russian) [CrossRef]

28. Martynyuk, A.A.; Stamov, G.; Stamova, I. Integral estimates of the solutions of fractional-like equations of perturbed motion. Nonlinear Anal. Model. Control 2019, 24, 138-149. [CrossRef]

29. Martynyuk, A.A.; Stamov, G.; Stamova, I. Practical stability analysis with respect to manifolds and boundedness of differential equations with fractional-like derivatives. Rocky Mt. J. Math. 2019, 49, 211-233. [CrossRef]

30. Ballinger, G.; Liu, X. Practical stability of impulsive delay differential equations and applications to control problems. In Optimization Methods and Applications. Applied Optimization; Yang, X., Teo, K.L., Caccetta, L., Eds.; Kluwer: Dordrecht, The Netherlands, 2001; Volume 52, pp. 3-21.

31. Bernfeld, S.R.; Lakshmikantham, V. Practical stability and Lyapunov functions. Thoku Math. J. 1980, 32, 607-613. [CrossRef]

32. Lakshmikantham, V.; Leela, S.; Martynyuk, A.A. Practical Stability of Nonlinear Systems; World Scientific: Teaneck, NJ, USA, 1990; ISBN 981-02-0351-9.

33. Martynyuk, A.A. (Ed.) Advances in Stability Theory at the End of the 20th Century. Stability and Control: Theory, Methods and Applications, 1st ed.; Taylor and Francis: New York, NY, USA, 2002; ISBN 0-203-16657-4. 
34. Stamova, I.M.; Stamov, G.T. Applied Impulsive Mathematical Models, 1st ed.; Springer: Cham, Switzerland, 2016; ISBN 978-3-319-28060-8.

35. Yang, C.; Zhang, Q.; Zhou, L. Practical stabilization and controllability of descriptor systems. Int. J. Inf. Syst. Sci. 2005, 1, 455-465.

36. Stamova, I.M.; Stamov, G.T. Functional and Impulsive Differential Equations of Fractional Order: Qualitative Analysis and Applications, 1st ed.; CRC Press, Taylor and Francis Group: Boca Raton, FL, USA, 2017; ISBN 9781498764834.

37. Wang, J.; Feckan, M.; Zhou, Y. A survey on impulsive fractional differential equations. Fract. Calc. Appl. Anal. 2016, 19, 806-831. [CrossRef]

38. Sitho, S.; Ntouyas, S.K.; Agarwal, P.; Tariboon, J. Noninstantaneous impulsive inequalities via conformable fractional calculus. J. Inequal. Appl. 2018, 2018, 261. [CrossRef]

39. Tariboon, J.; Ntouyas, S.K. Oscillation of impulsive conformable fractional differential equations. Open Math. 2016, 14, 497-508. [CrossRef]

40. Cicek, M.; Yaker, C.; Gücen, M.B. Practical stability in terms of two measures for fractional order systems in Caputo's sense with initial time difference. J. Frankl. Inst. 2014, 351, 732-742. [CrossRef]

41. Stamova, I.M.; Henderson, J. Practical stability analysis of fractional-order impulsive control systems. ISA Trans. 2016, 64, 77-85. [CrossRef] [PubMed]

42. Bernfeld, S.R.; Corduneanu, C.; Ignatyev, A.O. On the stability of invariant sets of functional differential equations. Nonlinear Anal. 2003, 55, 641-656. [CrossRef]

43. Bohner, M.; Stamova, I.; Stamov, G. Impulsive control functional differential systems of fractional order: Stability with respect to manifolds. Eur. Phys. J. Spec. Top. 2017, 226, 3591-3607. [CrossRef]

44. Smale, S. Stable manifolds for differential equations and diffeomorphisms. Ann. Scuola Norm. Sup. Pisa 1963, 3, 97-116.

45. Stamov, G. Lyapunov's functions and existence of integral manifolds for impulsive differential systems with time-varying delay. Methods Appl. Anal. 2009, 16, 291-298.

46. Liu, B.; Liu, X.; Liao, X. Robust stability of uncertain impulsive dynamical systems. J. Math. Anal. Appl. 2004, 290, 519-533. [CrossRef]

47. Stamov, G.T.; Alzabut, J.O. Almost periodic solutions in the PC-space for uncertain impulsive dynamical systems. Nonlinear Anal. 2011, 74, 4653-4659. [CrossRef]

48. Stamov, G.T.; Simeonov, S.; Stamova, I.M. Uncertain impulsive Lotka-Volterra competitive systems: Robust stability of almost periodic solutions. Chaos Solitons Fractals 2018, 110, 178-184. [CrossRef]

49. Aguila-Camacho, N.; Duarte-Mermoud, M.A. Boundedness of the solutions for certain classes of fractional differential equations with application to adaptive systems. ISA Trans. 2016, 60, 82-88. [CrossRef]

50. Ahmad, S.; Stamova, I.M. (Eds.) Lotka-Volterra and Related Systems: Recent Developments in Population Dynamics, 1st ed.; Walter de Gruyter: Berlin, Germany, 2013; ISBN 978-3-11-026984-0.

51. Gopalsamy, K. Stability and Oscillation in Delay Differential Equations of Population Dynamics, 1st ed.; Springer: Dordrecht, The Netherlands, 1992; ISBN 978-0-7923-1594-0.

52. Takeuchi, Y. Global Dynamical Properties of Lotka-Volterra Systems; World Scientific: Singapore, 1996; ISBN 978-981-02-2471-4, 978-981-4499-63-7.

53. Li, M.; Duan, Y.; Zhang, W.; Wang, M. The existence of positive periodic solutions of a class of Lotka-Volterra type impulsive systems with infinitely distributed delay. Comput. Math. Appl. 2005, 49, 1037-1044. [CrossRef]

54. Liu, X.; Rohlf, K. Impulsive control of a Lotka-Volterra system. IMA J. Math. Control Inform. 1998, 15, $269-284$. [CrossRef]

55. Liu, Z.; Wu, J.; Tang, R. Permanence and extinction of an impulsive delay competitive Lotka-Volterra model with periodic coefficients. IMA J. Appl. Math. 2009, 74, 559-573. [CrossRef]

56. Agrawal, S.K.; Srivastava, M.; Das, S. Synchronization between fractional-order Ravinovich-Fabrikant and Lotka-Volterra systems. Nonlinear Dyn. 2012, 69, 2277-2288. [CrossRef]

57. Stamov, G.; Stamova, I.M. On almost periodic processes in impulsive fractional-order competitive systems. J. Math. Chem. 2018, 56, 583-596. [CrossRef]

(C) 2019 by the authors. Licensee MDPI, Basel, Switzerland. This article is an open access article distributed under the terms and conditions of the Creative Commons Attribution (CC BY) license (http:/ / creativecommons.org/licenses/by/4.0/). 